\title{
Big Data, Internet of Things, Augmented Reality: technology convergence in visualization issues
}

\author{
D A Smuseva ${ }^{1}$, A Y Rolich ${ }^{1}$, L S Voskov ${ }^{1}$ and I Y Malakhov ${ }^{1}$ \\ ${ }^{1}$ National Research University "Higher School of Economics”, Myasnitskaya str., 20, \\ Moscow, Russia, 101000 \\ e-mail: dasmuseva@gmail.com, arolich@hse.ru,lvoskov@hse.ru
}

\begin{abstract}
The paper reviews the current situation of the Augmented Reality and Internet of Things markets. The implementing possibilities of AR for Big Data visualization from IoT devices are considered in this paper. The review and the analysis of methods, tools, products and data system of the visualization are presented. The paper provides an overview of the programs and devices of Augmented Reality, and an overview of development environments. The paper presents the existing classifications of computerized data visualization tools and proposes new classification, which takes into account interactive visualization, the purpose of the tool, the type of software product, the availability of ready-made templates, and other characteristics. The article proposes the architecture of the system for collecting data from IoT endpoint devices based on the Heltec modules. Experiments based on the developed experimental stand were carried out with Heltec devices of both versions to determine the number of losses with increasing distance between the sending device and the receiving device. The results of measuring the power consumption of these devices are presented in two modes: in standby mode and when sending a message to the Heltec endpoint device and in standby mode and when receiving a message for the base station. These studies were conducted using various data transfer protocols (LoRa, Wi-Fi and Bluetooth). The paper presents the result of the development of a digital twin of a university building and the development of augmented reality software for receiving data from real-time data collection devices.
\end{abstract}

\section{Introduction}

The introduction of new technologies is often focused on simplifying the routine actions of a person in everyday life. An example is the introduction of the Internet of Things technology, which requires an integrated approach to development that combines new technologies, skills and capabilities supported by effective management and organization. Such technology requires the support of user interaction and the digital ecosystem to enable the management of connected equipment. For this, it is necessary that the program can analyze and visualize the data obtained from the sensors. The relevance of this work lies in the fact that the two-dimensional visualization of data obtained from the sensors of the Internet of things, is not enough.

In this regard, there is a big growth in services for analyzing and visualizing the data received from the sensors. The novelty of the work lies in the fact that for the most part, such services represent information in a two-dimensional form. The problem is that programs of this format cannot ensure the full assimilation of information. With a large amount of data, the user may miss key points and, for example, in production, do not notice breakages due to clutter of the interface. 
Fortunately, nowadays modern technologies allow solving this problem with the help of augmented reality [1]. Consideration of the use of this technology in the field of IoT, it is possible to present its use in enterprises for timely identification of problems, in the home for remote control of equipment, in large production facilities for personnel training through the visualization of processes and instructions. In this regard, the purpose of this work is to identify methods, approaches and technologies for visualizing big data on the Internet of things using augmented reality.

\section{Big Data}

Over the past two decades, technological advances have led to a huge increase in data volumes. The term Big Data was coined for working with such data streams. This type of data is not only characterized by a large volume, but also demonstrates other unique characteristics compared to traditional data. For example, Big data is usually unstructured and may require real-time analysis. That is why for working with big data many new systems were created for collecting, transmitting, storing and processing large-scale data.

Due to the great popularity and diversity of Big Data technology, today there is no generally accepted definition for this term. In essence, big data means not only a large amount of data, but other functions that distinguish it from the concepts of "massive data" and "very large data". In the literature, you can find many definitions for Big data, consider three of them, which well explain the essence of the term Big data.

One of the currently popular definitions appeared back in 2001, in the report of META Group analyst (now Gartner) Dag Lani [2], who noted the equivalence of data management issues in three aspects $(3 \mathrm{Vs})$ :

- Volume. Big data is very large, often in petabytes or more. Examples include user data on Facebook, the flow of people on the subway or transactions using credit cards;

- Velocity. Big data is often generated continuously, often even in real time. For example, Twitter messages, cell phone locations, weather and air quality sensors;

- Variety. Big data can be of any type consisting of numbers, text, photos, video, audio and other data. Therefore, Big Data analysis must be flexible enough to deal with any type of data and be able to combine them.

Big data has three varieties: structured, semi-structured, and unstructured. Traditional data is usually structured and therefore easy to use and can be easily stored. However, the vast majority of today's data from sources such as Facebook, Twitter, YouTube and other user-generated content is unstructured. The high rate of receipt of large data means that data sets should be analyzed at a rate corresponding to the speed of data production.

Today, Big data can be found in many areas [3,4]. One of the most common ways to get big data is to collect data from sensors and devices. The popular branch of the development of this technology is the Internet of Things (IoT). It implies a global collection of all indicators from several sensors simultaneously (often in real time), the compilation of these data and their analysis.

\section{Internet of Things}

The Internet of Things (IoT) provides a network for connecting people, things, programs and data over the Internet to provide remote control and interactive implementation of services. Today, the amount of mobile devices with Internet connection exceeds the number of people on Earth. Moreover, in 2015, Gartner predicted [5] that by 2020 about 20.8 billion smart devices will be connected to the IoT. In order to support the work of devices connected to the IoT, it is necessary to have a special Internet of Things support service. Such IoT services need to collect, analyze and process information about sensors, they must have an interface for the operational management of information. The data collected from IoT devices can be impressive in size, since the number of sensors on the Internet of Things is very large. Therefore, a platform that can collect and store a huge amount of information from sensors is needed to 
control IoT. Such a platform should include IoT databases where cloud computing support is needed. Moreover, IoT data analysis and Big Data support is required.

The economic effect can be seen where the connection between the machines (hereinafter - M2M) is becoming increasingly important. However, person-to-person (hereinafter - P2P), person-to-machine (hereinafter - P2M) and machine to person (hereinafter - M2P) connections still remain the majority of the economic value of IoT. In the table below you can see the comparison of M2M with M2P or P2M, as well as with P2P technology.

IoT is used in various fields, consider some of them:

- Security. In this area, IoT is used to monitor alarms, monitor and control surveillance systems in real time and track people [6];

- Transport sphere. Intelligent applications for transportation systems are used for fleet management, road safety, emissions control, toll payments, real-time traffic monitoring, etc;

- Health. In this area, IoT is used for e-health, which includes individual health systems based on sensors for the human body;

- Utility management. The Internet of Things allows you to measure, prepare, and bill for gas, water, electricity, and more;

- Production. Production control is carried out through the monitoring and automation of the product chain;

- The scope of services. Control of supply and distribution, vending machines can also be monitored, and support can be provided in managing and providing services;

- Management of funds. Campus, building and home automation can be reached using IoT technology.

\section{Augmented Reality}

Augmented Reality (AR) is a technology that allows a user to real-time overlay virtual text or computer-generated images onto objects in the real world. Recognition occurs, usually with the help of a camera, and the output of augmented reality through the user's screen, telephone, monitor, helmet or Augmented Reality glasses, window or windshield of the car. Virtual text or image can be superimposed on the selected objects in the environment surrounding the user, to display additional information about the selected object.

It is customary to use two basic definitions of augmented reality. In 1994, Paul Milgram described the continuum "virtuality-reality" [7], i.e. the space between reality and virtuality, between which are Augmented Reality and augmented virtuality (figure 1). The real environment and the virtual environment unite in a mixed reality, where, in turn, augmented reality is based more on reality, and augmented virtuality on virtuality. Thus, Augmented Reality is the result of adding virtual objects to the number of objects perceived by man as elements of the real world.

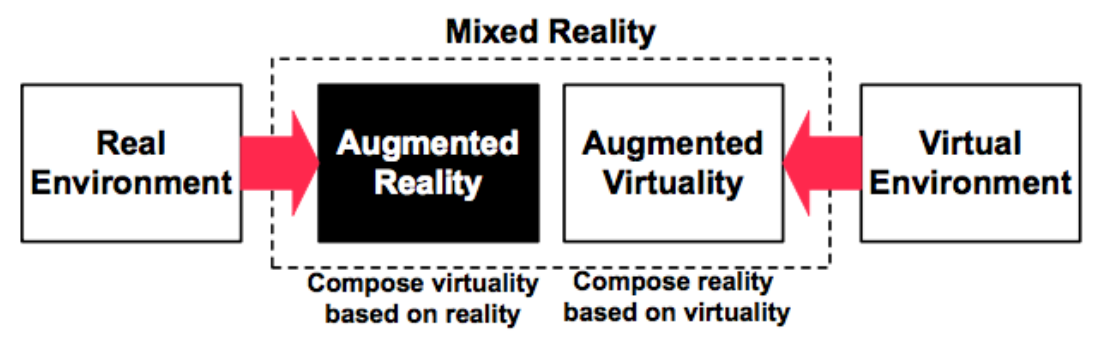

Figure 1. Milgram definition.

Augmented reality is based on context-sensitive computations. Thanks to this technology for the user, the real world and virtual objects coexist on the same view. For example, this technology can be applied to museums. If you point the camera of a smartphone with a special application of augmented reality on any exhibit, information about it will be displayed next to the exhibit. The user of augmented reality can 
receive useful information about a location or objects and can interact with virtual content in the real world.

\section{Data visuialization methods}

\subsection{Classification}

There are many computer visualization tools that allow you to convert large amounts of abstract data into formats that are effective for perception, cognition and communication. For example, in the work [8] the following classification of computer visualization tools is given:

1) Tool functionality: user (professional or beginner), teamwork support (how many people can simultaneously develop a model), extensibility (possibility of making changes), scalability.

2) Methods (characteristics of the methods used): number of methods, method type.

3) Task (characteristics of the problem to be solved): type of information (quantitative, qualitative), subject area (science, business, education)

4) Manufacturer (characteristics of the product): manufacturing-firm: (domestic, foreign), type of product (commercial, training and demonstration, conditionally free)

This classification considers the functionality of the tool from the point of view of user experience: the level of user skills, support for teamwork, extensibility and scalability. The availability of readymade templates, interactive visualization and other important characteristics are not taken into account in this work. The division of instrument manufacturers into domestic and foreign ones can be replaced by the multi-lingual nature of the proposed interfaces.

Consider the following classification of visualization and data analysis tools [9], the following classification of visualization and data analysis tools is proposed:

1) Category: analysis and charting, PC application, geographic information systems (GIS), library, network analysis, statistical analysis, time data analysis, data retrieval, data cleansing, data conversion, application/service for visualization and analysis, word clouds.

2) Multiple user support: true or false.

3) Data display on the map: true or false.

4) Platform.

5) User skill level: users who can work with spreadsheets, partially advanced users who are not afraid to spend two hours learning a new application, advanced users, users with programming experience or specialized knowledge in the field of GIS or network analysis.

6) Storage and processing location: user device, external server, public external server.

7) Posting results to the web: yes, with plugin, as a picture, no.

The considered classification summarizes such phenomena as a PC application, library, network analysis, statistical analysis and a word cloud. However, a PC application and a library are types of software, network analysis and statistical analysis are the purpose of the tool, and the word cloud is a data visualization method.

We have developed a new improved classification of computer visualization tools that expands and improves the previously proposed options:

1) Purpose: general purpose; specialized (GIS, business intelligence tools, network analysis); auxiliary (designed to prepare data for visualization).

2) User skill level: beginer; intermediate; advanced; user with programming experience or special knowledge.

3) Software: programming language (R, Python); library (D3, ChartJS, Highcharts JS, Raphaël и др.); application/service.

4) Platform.

5) Distribution: commercial; conditionally free; free.

6) Multilingual interface: yes; no.

7) Need to register: yes; no.

8) Multiplayer mode: yes; no.

9) Types of visualized data: qualitative; qualitative and quantitative; quantitative. 
10) Supported Databases and file formats.

11) Availability of ready-made templates: yes; no.

12) Interactive visualization: yes; no.

13) Storage and processing location: user device; external server; public external server.

14) Formats of export results.

This classification takes into account interactive visualization, the purpose of the tool, the type of software product, the availability of ready-made templates, and other characteristics.

\subsection{Analysis of Big Data visualization methods}

The aggregated data from all devices connected to the IoT network must be correctly reproduced for the decision maker. For these purposes, visualization is a suitable tool. Data visualization represents data in some systematic form, including attributes and variables for a unit of information. Visualization approaches are used to create tables, charts, images, and other intuitive ways to display data. Big data visualization is not as simple as traditional small data sets [10]. The expansion of traditional approaches to visualization has already appeared, but far from enough. With large-scale data visualization, many researchers use feature extraction and geometric modeling to significantly reduce the size of the data before actually rendering the data. Choosing the right data representation is also very important when visualizing big data [11].

Often, standard methods of data visualization are used, which include such methods as a table, histogram, scatter plot, line diagram, pie chart, block diagram, bubble chart, combination of charts, time line, entity relationship diagram, etc. In addition, the standard methods of data visualization include additional methods: parallel coordinates, treemap, cone-shaped tree, semantic network, etc [10]. Parallel coordinates (figure 2) are used to construct individual data elements in many dimensions. This method of data visualization is very useful when displaying multidimensional data [12].

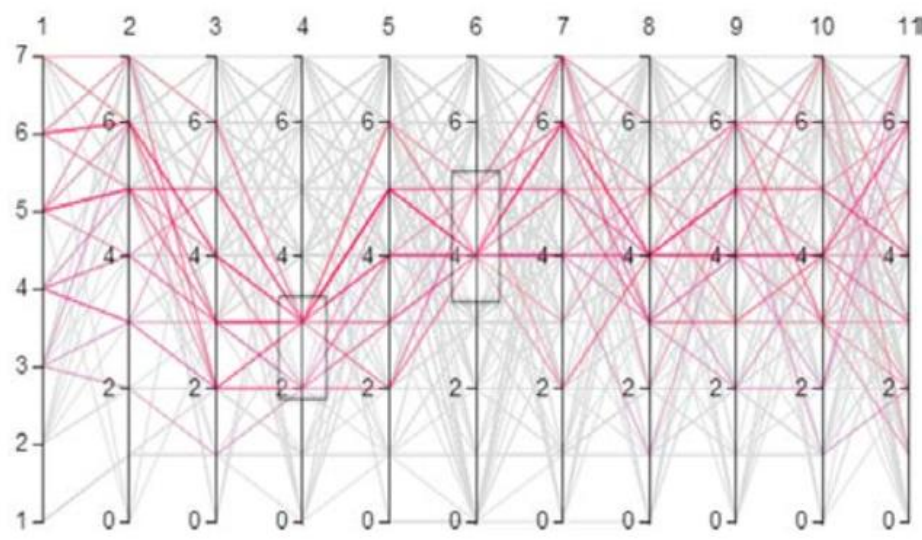

Figure 2. Parallel coordinates.

Treemap (figure 3) - an effective method of visualizing the hierarchy, which is a map, divided into sections. The smallest rectangles represent one measure, while color is often used to represent another measure of data. Figure 3 shows an example of a treemap collection set for streaming music and video tracks in a social networking community [13].

A cone-shaped tree is another method that displays hierarchical data, such as an organizational body in three dimensions. Branches grow in the form of a cone. The semantic network is a graphical representation of the logical connection between different concepts. It generates a directed graph, a combination of nodes or vertices, edges or arcs, and an inscription above each edge [10]. Visualization is not only static, but also interactive, thanks to such approaches as scaling (zooming in and out); review and detail; pan; as well as focus and context or fisheye [10]. Below are the steps for interactive visualization:

- Choice. This implies an interactive selection of data objects, a subset, parts of whole data, or the entire data set in accordance with the interests of the user; 
- Linking: useful for linking information between several types of graphs. An example is shown in figure 4;

- Filtering: helps users adjust the amount of information to display. It reduces the amount of data and focuses on information of interest;

- Regrouping or reuse: since spatial layout is the most important visual display, rebuilding the spatial layout of information is very effective for creating different ideas;

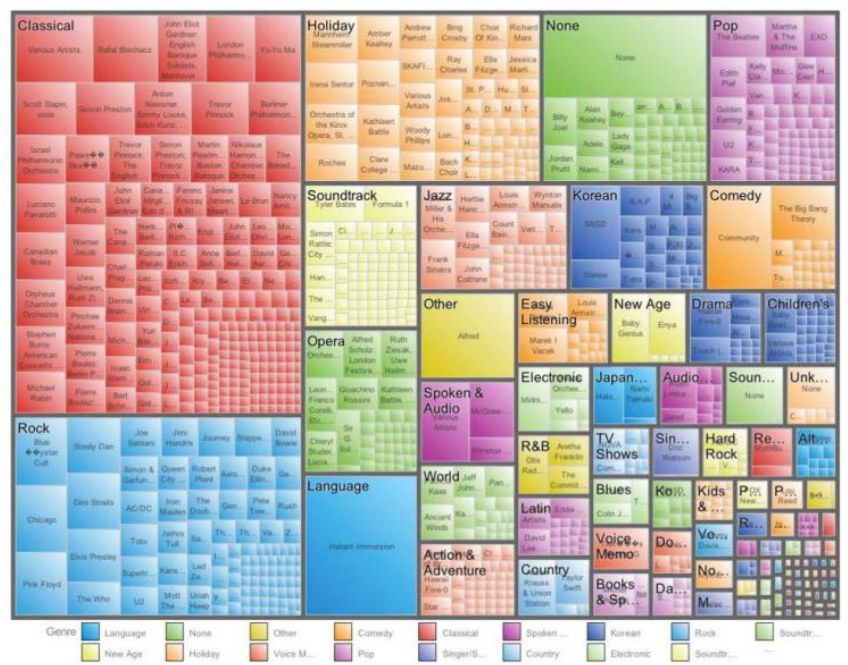

Figure 3. Analysis of musical compositions using a treemap.

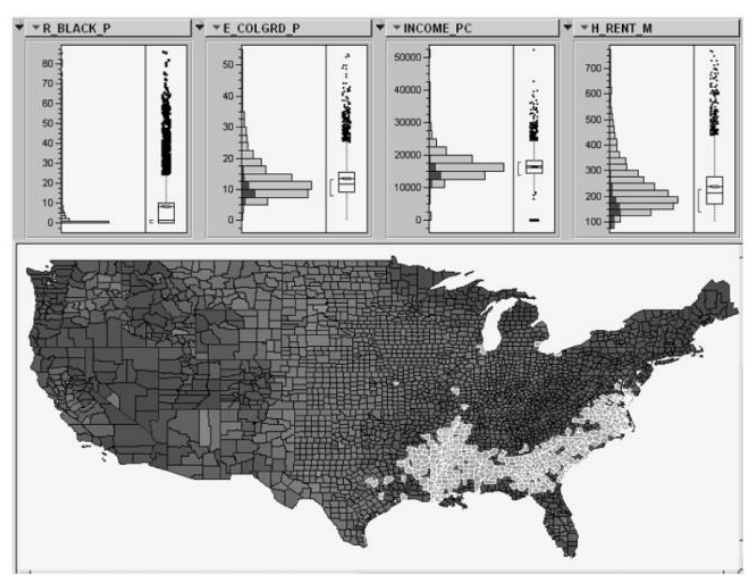

Figure 4. Interactive linking between histogram graphs and a geographic map.

The scalability and dynamics inherent in big data are the main problems in visual analytics. Big data visualization with diversity and heterogeneity (structured, semi-structured, and unstructured) is a big problem. As described above, speed (Velocity) is one of the important factors for analyzing Big Data. Cloud computing and an advanced graphical user interface can be combined with big data to better manage the scalability of big data [14].

Big data often has an unstructured data format, so due to bandwidth limitations and power requirements, the visualization must fit the data in order to efficiently get up-to-date information. Because of the large size of the data, there is a need for parallel computations, the task of which is to distribute computations for independent tasks that can be performed simultaneously [15].

The following problems exist in Big Data visualization [16]:

- Visual noise. Most of the objects in the dataset are too relative to each other. Users cannot separate them as separate objects on the screen; 
- Loss of information. To simplify the calculations, it is often necessary to reduce the data sets, which leads to loss of information;

- Complicated image perception. Data visualization methods are limited not only by the aspect ratio and resolution of the device from which the visualization is viewed, but also by the physical limitations of perception;

- High speed image change. Users observe the data and cannot respond to the amount of data changes or their intensity on the display;

- High performance requirements.

Visualizing each data point can overwhelm the schedule and can suppress users' perceptions and cognitive abilities, and reducing data by sampling or filtering can lead to a loss of interesting emissions and deviations. Requesting large data warehouses can lead to high latency, disrupting real-time interaction [17].

Ways of solving problems of Big Data visualization [18]:

1. Satisfying the need for speed. This implies improved hardware with increased memory and powerful parallel processing,

2. Understanding the data. One solution is for people who receive data to have relevant experience and knowledge for quick understanding,

3. Solving the problem of data quality. It is necessary to control that the data arriving at the visualization were cleared from unnecessary information,

4. Synthesis of information. One way is to combine the data into a higher-level view, where smaller groups of data are visible and the data can be effectively visualized,

5. Create exceptions for emissions. A possible solution is to remove outliers from the data or create a separate chart for outliers.

Big data can be visualized using a number of approaches and methods that were analyzed and classified (table 1) according to the following data criteria: large data support, support for different types of data, and the capacity to dynamically display data changes [7].

- Treemap: based on the visualization of spatial images of hierarchical data;

- Circle Packing: A direct alternative to treemap. However, it uses circles as a primitive form, which can also be included in circles from a higher level of hierarchy;

- Sunburst: uses a treemap visualization and converts it into a polar coordinate system. The main difference is that the variable parameters are not the width and height, but the radius and length of the arc;

- Parallel coordinates: extends visual analysis using a variety of data factors for different objects;

- Streamgraph: this is a type of graph of a stack region that is offset around a central axis, which results in a smooth and organic form;

- Circular Network Diagram: The data object is placed around the circle and connected by curves based on the level of their interconnections. Different line widths or color saturation are commonly used to measure the coherence of an object.

Table 1. Analysis of big data visualization methods.

\begin{tabular}{lccc}
\hline & $\begin{array}{c}\text { Large amount } \\
\text { of data }\end{array}$ & $\begin{array}{c}\text { Variety } \\
\text { of data }\end{array}$ & $\begin{array}{c}\text { Data } \\
\text { dynamics }\end{array}$ \\
\cline { 2 - 4 } Treemap & + & - & - \\
Circle Packing & + & - & - \\
Sunburst & + & - & +
\end{tabular}

Many data visualization tools work on the Hadoop platform. Such modules in Hadoop are: Hadoop Common, Hadoop Distributed File System (HDFS), Hadoop YARN, and Hadoop MapReduce. There 
are also separate softwares, but they all represent a two-dimensional visualization of data, which is a difficulty for the user to understand the information. The solution to this problem is to use augmented reality as a visualization tool, which will facilitate the work of the user and allow you to visualize the data in a convenient form.

\section{Big Data, IoT and AR: technology convergence in visualization issues}

In 1979, a major accident at a nuclear power plant occurred in the United States, the cause of the accident was erroneous actions by personnel who did not understand the incoming data and did not identify the problem in time [12]. The introduction of Augmented Reality in the visualization of data collected from IoT-sensors, will make work in many areas more secure and effective. The use of this technology will allow real-time monitoring of the indicators of the sensors, output the data in a visual form, it is also possible remote user interaction with the equipment. Thus, not only the working personnel who are in close proximity to the equipment can make an inspection, but also the manager will be able to more accurately control the production situation with the help of installed smart sensors in all rooms. If something goes wrong, workers can instantly fix the problem, or transmit the data to engineers outside the building.

Also, this technology can be used for training and staff training. Companies can develop interactive manuals in augmented reality, filling them with interactive $3 \mathrm{D}$ equipment models and visual step-by-step instructions. For example, global manufacturer Caterpillar Inc. makes good use of AR to train its engineers and makes them more efficient. Engineers can use AR glasses or a smartphone to get virtual, step-by-step live instructions on how to efficiently perform machine repairs and other maintenance tasks. Many examples can also be seen in the areas of health, education, aviation and space, travel, real estate and other sectors.

The interaction of AR and IoT requires a large number of calculations; in addition, the volume of processing of augmented reality directly affects the power consumption of the device and its lifespan. That is why to work with AR on smartphones an additional cloud database is needed to reduce the load, save energy and overcome the limitations of the phone's memory. It is worth noting that the use of cloud technologies should be controlled by adaptive management, since the transfer of all computational processes to the cloud platform can lead to large delays in the operation of the program. To use cloud resources efficiently, you need to monitor the cloud server load and network congestion status so that they are balanced. It is necessary to monitor the state of the network, the status of the cloud server, the energy status of the device and the quality of perception (QoE, Quality of Experiance).

Using an AR application on smartphones, tablets, or corporate AR headsets, workers can monitor connected equipment to identify problems and perform maintenance. AR headsets, such as Google Glass Enterprise and Microsoft HoloLens, can also be useful in enterprises, displaying stepby-step online training and tutorials for assembling and operating equipment that would otherwise require reading thick technical manuals and instructions.

To create an Augmented Reality program with the ability to visualize data from the IoT sensors, you can use the existing augmented reality SDKs: ARToolkit [19], EasyAR [20], OpenCV [21], Maxst [22], Kudan [23], Catchoom On-Device Image Recognition SDK [24] and Vuforia SDK [25]. With the help of such SDKs, only a part with augmented reality can be developed, but additional programs will be needed to connect IoT devices and collect data. For the productive implementation of programs, there are ready-made solutions that combine all stages of program development, including connecting sensors and visualizing data. The main leaders among the platforms for creating programs with AR for the Internet of Things are PTC, Augmenta, Bosh, Reflect.

Currently, the main area of convergence of the listed technologies is the visualization of big data coming from IoT-devices in real time on mobile devices with augmented reality function. Conventionally, we can distinguish the following types of convergence:

1. Visualization of sensor data and object management.

2. Monitoring equipment performance and decision support systems. The information presented in a visual form will allow timely troubleshooting equipment problems and improving the quality of 
manufactured products. Ideally, this technology should be implemented at every stage of production.

3. People will be able to interact with electronics using 3D interfaces, which raises new questions about the energy efficiency of mobile devices, which are displays for data visualization (smartphones, tablets, smart watches or AR helmets).

The current trends in the field of the Internet of Things are the creation of new hardware platforms, the development of specialized operating systems, the development and optimization of application layer protocols, and the development of models and methods for data visualization aimed at improving the energy efficiency of the Internet of Things, including mobile devices, to increase battery life. The lack of mathematical models, methods and tools for organizing dynamic reconfiguration of graphical interfaces of IoT devices (including using the AR) is a serious obstacle for further research, as well as for increasing the time for effective functioning of mobile devices and networks of software systems with limited resources. In this regard, the task of research and development of an integrated model that makes it possible to assess the efficiency of energy consumption and other limited resources of mobile devices, methods that make it possible to optimize the consumption of resources of a mobile device according to the criterion of maximizing residual energy taking into account the limitations and requirements of users for final applications and graphic interfaces, and means of organizing reconfiguration of graphic interfaces of devices.

\section{Experimet and technology convergence example}

To demonstrate the process of convergence of technologies we used software for iOS. This software allows you to visualize data from sensors of various devices, combined in a data collection system, using augmented reality. The experimental data acquisition system (figure 5) consists of endpoint devices that can collect temperature, humidity, light, noise and the presence of movement data. Each end device sends data using one of the LoRa, Wi-Fi or Bluetooth protocols to the base station, which generates data messages and sends them to the ThingWorx cloud platform. Heltec LoRa 32 (V2) [26] microcontrollers were used as the main components for receiving and transmitting data from various sensors. Most of them served as endpoint devices that processed and prepared for sending messages containing indicators from the sensors. While the other part was used as a basic device for receiving messages from all end sensors. For the base station organization, the Raspberry PI 3 single board computer was used. This computer is a ready-made solution for working with low-resource programs. The approach that was chosen to work with this computer allows you to use it as a full-fledged server without using external interfaces and communicating and sending commands to the Raspberry only over an SSL connection. With the help of this computer, it became possible to store data coming from devices with sensors on the Raspberry, thus turning it into a machine with the ability to send data for services, including applications, using a special network broker.

Mosquitto is used as an MQTT broker. It allows you to send mqtt messages to both cloud platforms and directly to applications. This is done because MQTT is an inter-computer connection (M2M) protocol, in other words, for the Internet of things. It was designed as an extremely easy way to publish publish (subscribe) and subscribe. This is useful for connections to remote locations where a small amount of code and high bandwidth are required. For example, it is widely used in sensors that communicate with a broker through satellite communications, through dial-up connections with health care providers, and in a number of home automation scenarios. It is also ideal for mobile applications because of its small size, low power consumption, minimized data packets and efficient distribution of information to one or several recipients. MQTT broker is used as follows: we form a request in Unity MQTT, which is needed in order to get data from the cloud platform. When the data on the platform changes, the broker sends the data with changes. MQTT broker and Unity are connected in publishersubscriber mode. The software subscribes to receive data from the broker's MQTT and polls it with a given frequency. 


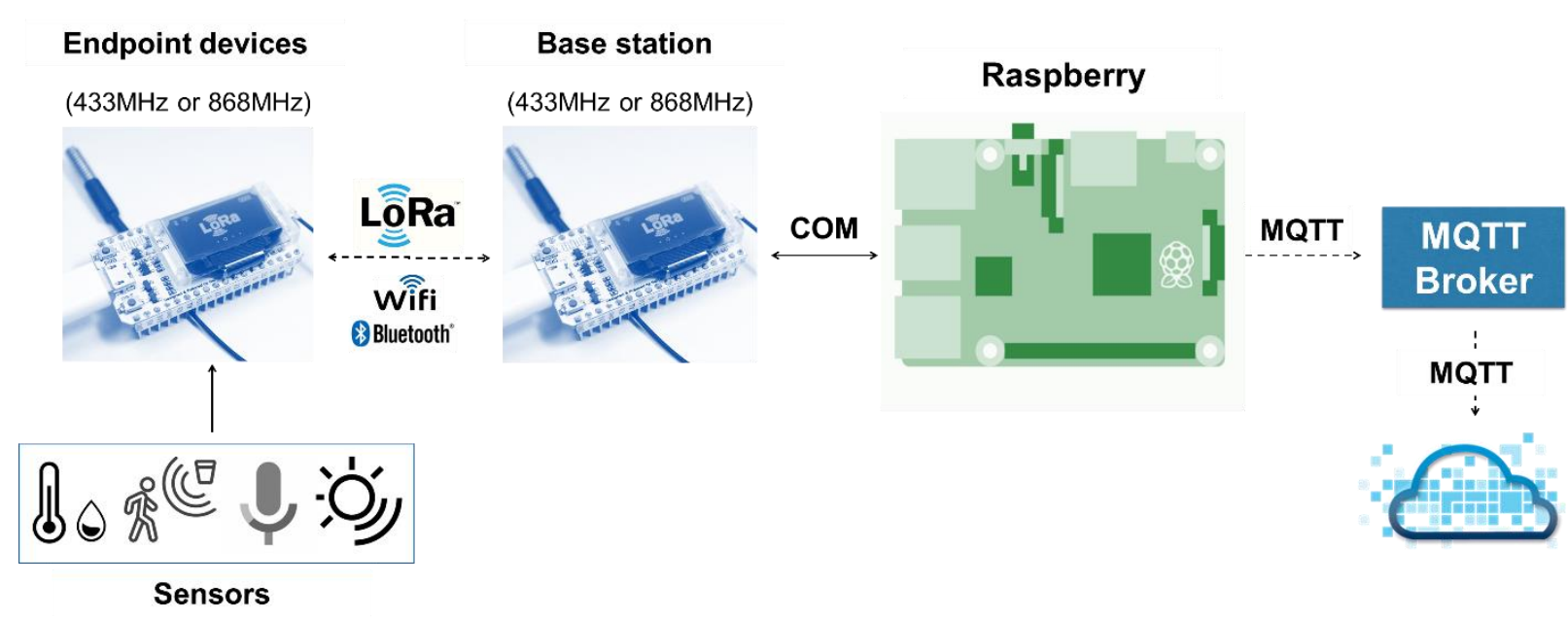

Figure 5. Data acquisition system architecture.

The developed experimental stand allowed us to analyze both versions of Heltec devices for the number of losses when the distance between the sender device and the receiver device was increased, to measure the power consumption of these devices in two modes: during standby and while sending a message for the final Heltec, but also during waiting time and while receiving a message for the base station. These studies were conducted using LoRa, Wi-Fi and Bluetooth, for each type of device, the average operating time per battery charge will be calculated.

The first test involves the use of two devices, one receiver, it is in a stationary position, and one transmitter, and it must be transferred, thereby increasing the distance between these devices. The results of the experiment are presented in table 2. As can be seen from the information received, regardless of the version and the Heltec clock frequency, up to 40 meters from each other, all devices work with $100 \%$ packet delivery, which means that no packet was lost for the tested period. After increasing the distance between devices by 10 meters more, the number of undelivered packages in all cases began to grow, but the percentage of packets received is still very high, not a single value has dropped below 95\%. After the next 2 iterations of the increase in the distance between the Heltec modules, the number of lost packets increases by about 3 and 4 times, respectively. At the same time, it becomes obvious that devices of the second version have a preponderance of losses in the direction of the lower frequency, 9 packets against 5 at 60 meters and 16 against 10 at 70 meters. With a slight advance, the best packet delivery percentage is at Heltec LoRa v2, operating at a frequency of 868 $\mathrm{MHz}$. Thus, it was revealed that, at long distances, priority is given to using the device second versions with a clock frequency of $868 \mathrm{MHz}$.

In the second experiment, an analysis of the current consumption of the endpoint devices was carried out using various data transfer protocols. This experiment allows you to check the actual current consumption by the Heltec endpoint devices with the payload on the microcontroller in the form of sending packets using various data transfer interfaces. There is no intentional distinction here between the versions of the Heltec modules, since both versions consume almost the same amount of current, so the separation of tests by versions has no scientific basis [27]. Table 3 presents data from tests of current consumption by devices in two modes: waiting for polling and sending a packet. As you can see from the data, as expected, the highest energy efficiency was achieved using the LoRa interface. The second place in terms of efficiency of current consumption was taken by the protocol for Bluetooth communication, while Wi-Fi was in last place in terms of current consumption both in data transfer mode and in standby mode. In this case, the relationship between the readings of the end device and the base station is traced, so the base station consumes on average about 35\% more current than the end device in standby mode (polling). In this case, the difference between the energy indices for the transmission (reception) modes is about one tenth of the consumption of the Heltec end device. 
Table 2. Dependence of the number of delivered Heltec packages on the distance.

\begin{tabular}{lccc}
\hline & Heltec LoRa v1 & Heltec LoRa v2 & Heltec LoRa v2 \\
\hline Distance / Frequency & $\mathrm{MHz}$ & $\mathrm{MHz}$ & $\mathrm{MHz}$ \\
\cline { 2 - 4 }$>40$ meters & $100 \%$ & $100 \%$ & $100 \%$ \\
50 meters & $96,6 \%$ & $95 \%$ & $96,6 \%$ \\
60 meters & $90 \%$ & $85 \%$ & $91,6 \%$ \\
70 meters & $81,6 \%$ & $73,3 \%$ & $83,3 \%$ \\
\hline
\end{tabular}

To demonstrate the convergence process of the technology IoT and AR was used Vuforia. The key factor in choosing was the maximum distance to capture and hold the marker. The most important thing is that Vuforia has integration with the Thingworx cloud platform, in which the data collection system is connected. The software has two modes of operation: identification of endppont devices and data output in augmented reality (figure 6) or the output of a virtual digital twin of the building in which the data acquisition system is located (figure 7), and data output in different parts of this digital twin. All data that comes from the endpoint devices in the form of text comes to the 3D rendering unit along with 3D models, in which information about the properties of the models, textures, materials and illumination is processed. In the first mode of operation, the camera compares the received frames with the base of markers. As soon as the similarity is found, a request for a cloud platform is formed, a response from the broker's MQTT comes and then digital content is output. In the second case, using the camera, it is necessary to scan the surrounding space to create a mesh grid to which the virtual digital twin of the building can be attached.

Table 3. Dependence of power consumption of the endpoint data collection device and the base station on the operating mode used.

\begin{tabular}{lrrrr}
\hline & \multicolumn{2}{c}{$\begin{array}{c}\text { Endpoint data collection } \\
\text { device }\end{array}$} & \multicolumn{2}{c}{ Base station } \\
\hline $\begin{array}{l}\text { Protocol / Operating } \\
\text { mode }\end{array}$ & Standby & & & \\
\cline { 2 - 5 } & & & & \\
LoRa & $80 \mathrm{~mA}$ & $170 \mathrm{~mA}$ & $130 \mathrm{~mA}$ & $180 \mathrm{~mA}$ \\
Wi-Fi & $170 \mathrm{~mA}$ & $300 \mathrm{~mA}$ & $240 \mathrm{~mA}$ & $330 \mathrm{~mA}$ \\
Bluetooth & $130 \mathrm{~mA}$ & $230 \mathrm{~mA}$ & $200 \mathrm{~mA}$ & $270 \mathrm{~mA}$ \\
\hline
\end{tabular}

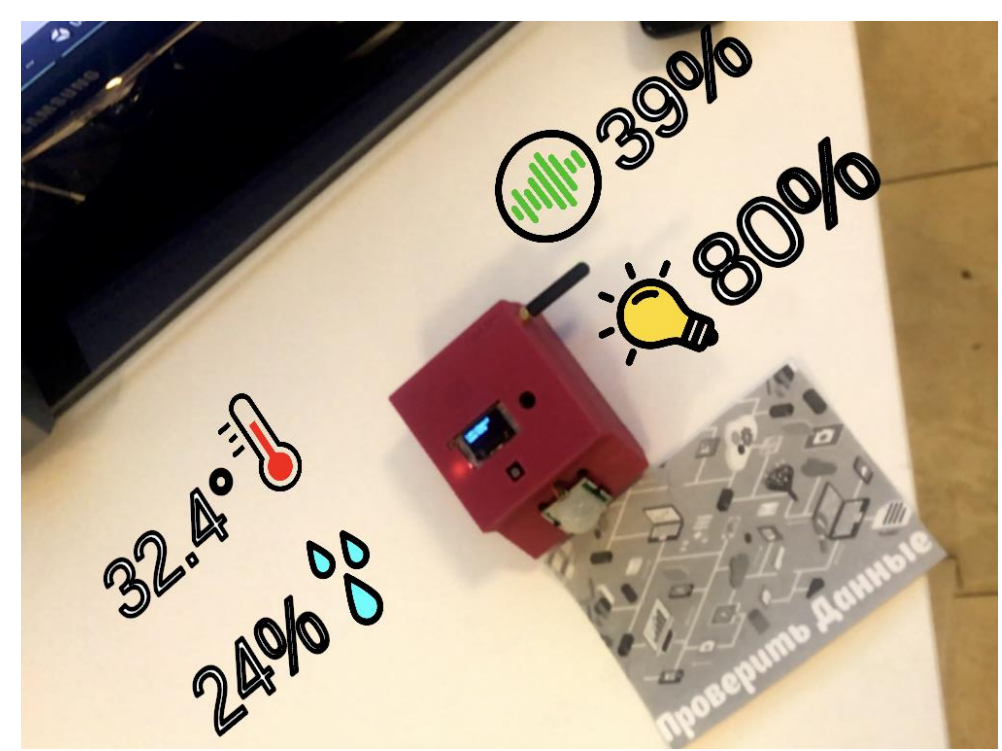

Figure 6. Endpoint device and data vusualization using AR. 

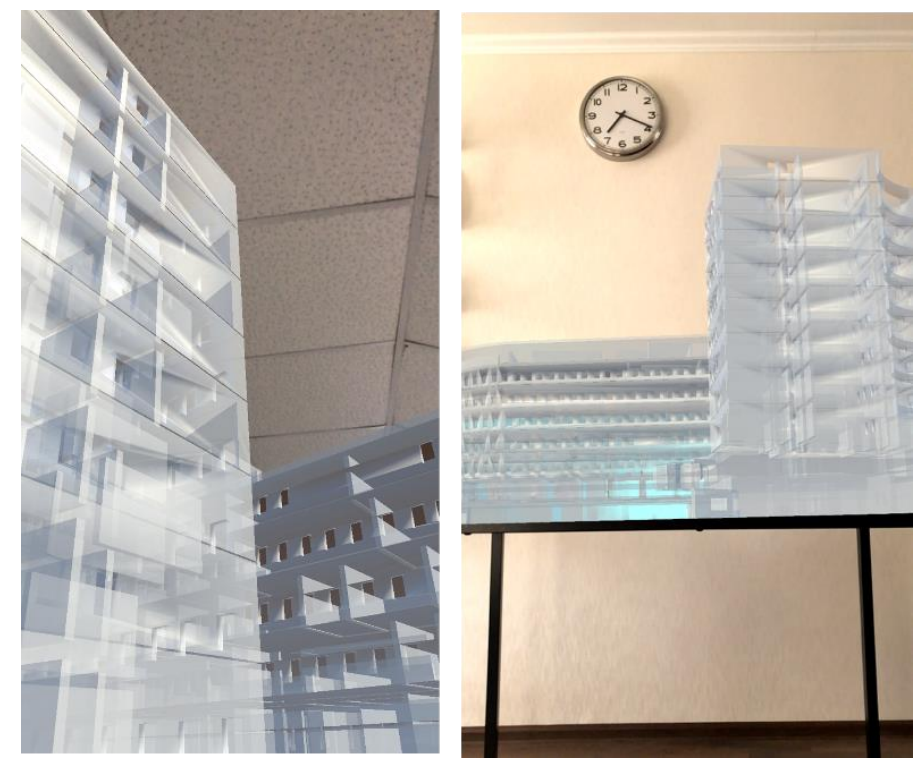

Figure 7. Digital twin of the building in which the data acquisition system is located.

\section{Conclusion}

The connenction of AR with the IoT ecosystem presents tremendous opportunities for various industries. Using this approach can make machines smarter by integrating sensors and intelligent systems that can monitor and produce data to solve problems in a timely manner and prevent downtime and breakdowns. IoT platforms provide a scalable, flexible, and secure way to cost-effectively extend AR applications. The use of such programs will allow companies to remotely diagnose and fix problems, preventing equipment and system system failures before they occur. This predictive service not only eliminates system downtime, but also optimizes energy use, which saves money. This paper proposes a classification of computer-based data visualization tools that can be used in developing a decision support system in the data visualization field.

To demonstrate the potential convergence of technology, an experimental data collection system stand was developed. The proposed data collection system allowed us to conduct experiments to determine the dependence of the data transmission distance in the building of the university on the use of specific models of Heltec modules of various versions and at different frequencies. Experiments were carried out to measure the energy consumption of data collection endpoint devices based on Heltec modules. To visualize data coming in real time from elements of the data collection system, software was developed using the Vuforia library, which allows visualizing the digital twinof the university building and all the information from the data collection system.

\section{References}

[1] Agogi E 2011 Augmented Reality in Education Athens, Greece: EDEN-2011 Open Classroom Conference 1-88

[2] Laney D $20013 d$ data management: Controlling data volume, velocity and variety (Gartner, Stamford, CT, USA, White Paper)

[3] Rytsarev I A, Kirsh D V and Kupriyanov A V 2018 Clustering of media content from social networksusing bigdata technology Computer Optics 42(5) 921-927 DOI: $10.18287 /$ 2412-6179-2018-42-5-921-927

[4] Agafonov A A, Yumaganov A S and Myasnikov V V 2018 Big data analysis in a geoinformaticproblem of short-term traffic flow forecasting based on a $\mathrm{k}$ nearest neighbors method Computer Optics 42(6) 1101-1111 DOI: 10.18287/2412-6179-2018-42-6-1101-1111

[5] van der Meulen R Gartner URL: https://www.gartner.com/newsroom/id/3165317 (14.04.2018)

[6] Evsutin O O, Kokurina A S and Meshcheryakov R V 2019 A review of methods of embedding information in digital objects for security in the internet of things Computer Optics 43(1) 137-154 DOI: $10.18287 / 2412-6179-2019-43-1-137-154$ 
[7] Milgram P, Kishino A F 1994 Taxonomy of Mixed Reality Visual Displays IEICE Transactionson Information Systems 1321-1329

[8] Gavrilova T A, Gulyakina N A 2008 Visual methods of working with knowledge: an attempt to review Iskusstvennyj intellekt $i$ prinyatie reshenij 1 15-21

[9] Machlis Sh Chart and image gallery: 30+ free tools for data visualization and analysis URL: https://www.computerworld.com/article/2506820/business-intelligence/business-intelligence-chart-and -image-gallery-30-free-tools-for-data-visualization-and-analysis.html (18.04.2018)

[10] Khan M, Khan S S 2011 Data and Information Visualization Methods and Interactive Mechanisms: a Survey International Journal of Computer Applications 1-14

[11] Chen C L P, Zhang C Y 2014 Data-intensive applications Challenges, Techniques and Technologies 314-347

[12] Porter B 2012 Visualizing Big Data in Drupal: Using Data Visualizations to Drive Knowledge Discovery Report, University of Washington 1-38

[13] Keahey T A 2013 Using Big Data Technical Report 1-16

[14] Sucharitha V, Subash S R and Prakash P 2014 Visualization of Big Data: Its Tools and Challenges International Journal of Applied Engineering Research 5277-5290

[15] Childs H, Geveci B, Meredith J, Moreland K, Sewell C, Bethel E W, Kuhlen T and Schroeder W 2013 Berkeley National Laboratory Joint Research Report, Oak Ridge National Laboratory Sandy National Laboratories, Los Alamos National Laboratory, RWTH Aachen University 1-11

[16] Gorodov E Y, Gubarev V V 2013 Analytical Review of Data Visualization Methods in Big Data Journal of Electrical and Computer Engineering 4 1-7

[17] Liu Z, Jiangz B and Heer J 2013 imMens: Real-time Visual Querying of Big Data Eurographics Conference on Visualization (EuroVis) 421-430

[18] SAS Institute Inc 2013 Survey data analyzers Report 1-2

[19] Azuma R T 1997 A survey of augmented reality Presence: Teleoperators and virtual environments 6(4) 355-385

[20] Open Source Augmented Reality SDK ARToolKit.org URL: https://artoolkit.org/ (22.04.2018)

[21] Easy AR SDK URL: http://www.easyar.com/ (12.05.2018)

[22] OpenCV library URL: http://opencv.org/ (15.05.2018)

[23] Maxst URL: http://maxst.com/image_ar/?_Lang=en (22.05.2018)

[24] Kudan Computer Vision Kudan limited URL: https://www.kudan.eu/ (22.04.2018)

[25] Catchoom - Image Recognition and Augmented Reality URL: https://catchoom.com (22.04.2018)

[26] Vuforia Augmented Reality URL: https://www.vuforia.com (22.04.2019)

[27] WiFi LoRa 32 (V2) URL: https://heltec.org/project/wifi-lora-32/ (22.04.2019)

[28] Fernández-Ahumada L M 2019 Proposal for the Design of Monitoring and Operating Irrigation Networks Based on IoT, Cloud Computing and Free Hardware Technologies Sensors 19(10) 2318

\section{Acknowledgments}

The publication was prepared within the framework of the Academic Fund Program at the National Research University Higher School of Economics (HSE) in 2019- 2020 (grant № 19-04-022) and by the Russian Academic Excellence Project "5-100". 